\title{
An Approach to Developing Multi-Tenancy SaaS Using Metaprogramming
}

\author{
Josino Rodrigues \\ CIn-UFPE, UFRPE \\ jrn4@cin.ufpe.br \\ Paulo Silveira \\ CIn-UFPE \\ pamsn@cin.ufpe.br
}

\author{
Andreza Leite \\ CIn-UFPE, UFRPE \\ ala4@cin.ufpe.br \\ Vinícius Garcia \\ CIn-UFPE \\ vcg@cin.ufpe.br
}

\author{
Júlio César Damasceno \\ CIn-UFPE, UFRPE \\ jcd@cin.ufpe.br \\ Silvio Meira \\ CIn-UFPE \\ silvio@meira.com
}

\begin{abstract}
In last years have seen an increase in SaaS (Software as a Service) use. The development of multi-tenancy web application (one of the main ways to provide SaaS) increased considerably after the start of call "Web 2.0 Age". This work presents an approach for implementation of a multitenancy SaaS application, and an architecture based on plugins and metaprogramming to achieve a high level software reuse. This approach is presented through an experience report described throughout the paper.
\end{abstract}

\section{Categories and Subject Descriptors}

D.2.8 [Software Engineering]: Software ArchitecturesPatterns

\section{Keywords}

Multi-Tenancy, SaaS, Metaprogramação, Software as a Service, Software como serviço, Arquitetura

\section{INTRODUÇÃO}

Segundo estudos da Gartner, na América Latina, a receita estimada para SaaS (Software as a Service) esta para alcançar U\$ 419,7 milhões até o final de 2012, valor esse que atingiu U\$ 331,1 milhões no ano anterior. Espera-se que Brasil e México conduzam a maioria das oportunidades de adoção e receita[5].

Segundo Harris e Ahmed [6] existem três tipos diferentes de aplicações SaaS:

- Multi-instance: executam em ambientes onde o servidor web é compartilhado. Nessa abordagem uma cópia da aplicação é inicialmente configurada e então implantada como um contexto do servidor web.

- Single-instance: as aplicações provêem serviços para um único cliente e executam em um servidor exclusivo. Essa pode ser considerada a abordagem com maior

Permission to make digital or hard copies of all or part of this work for personal or classroom use is granted without fee provided that copies are not made or distributed for profit or commercial advantage and that copies bear this notice and the full citation on the first page. To copy otherwise, to republish, to post on servers or to redistribute to lists, requires prior specific permission and/or a fee.

WebMedia'12, October 15-18, 2012, São Paulo/SP, Brazil.

Copyright 2012 ACM 978-1-4503-1706-1/12/10 ...\$15.00. desperdício de recursos, dado que um servidor pode executar com apenas $10 \%$ de sua capacidade [6].

- Multi-tenancy: provê uma única aplicação compartilhada por vários clientes. Nessa abordagem várias aplicações "virtuais" (tenants) são criadas na mesma instância de software.

Arquiteturas multi-tenancy tem sido identificadas como uma vantagem competitiva potencialmente crítica sobre as arquiteturas clássicas single-tenant em certos domínios[4]. Contudo desenvolvedores de software têm dificuldade em projetar e implementar arquiteturas multi-tenancy eficientes[9] devido à falta de documentação e formalização do entendimento dos conceitos arquiteturais.

O objetivo desse trabalho é apresentar uma experiência prática obtida durante a adoção da arquitetura multi-tenancy, de forma a auxiliar desenvolvedores e engenheiros de software no processo de desenvolvimento de SaaS. Esse trabalho está organizado da seguinte forma: a Seção 2 apresenta o problema solucionado durante a implementação da aplicação multi-tenancy, a Seção 3 apresenta a metodologia utilizada nesse trabalho, a Seção 4 descreve a escolha da arquitetura e sua implementação, por fim, a Seção 5 apresenta as conclusões e trabalhos futuros.

\section{PROBLEMA}

O problema a ser solucionado surgiu da necessidade de desenvolver um software para suporte ao desenvolvimento de LPS (Linha de Produtos de Software) que fosse executado no modelo de software como serviço. Esse aplicativo foi desenvolvido para atender a necessidade da empresa Rise ${ }^{1}$, onde ocorreu uma residência em reuso de software na qual os residentes deveriam definir e documentar várias LPS para diferentes empresas.

Cavalcanti et al. [3] apresentam um metamodelo que tem como objetivo coordenar as atividades relacionadas ao desenvolvimento de uma LPS, pelo gerenciamento de suas diferentes fases e da manutenção da rastreabilidade e variabilidade entre os diferentes artefatos. Esse metamodelo foi representado utilizando a notação UML e divide-se em cinco sub-modelos: Gerenciamento de Projeto, Gerenciamento de Risco, Escopo, Requisitos e Testes. A descrição de cada submodelo pode ser encontrada em [3].

Além dos requisitos citados acima, ainda existiam alguns fatores que poderiam influenciar nas decisões de projeto:

- A aplicação seria inicialmente provida como SaaS para

\footnotetext{
${ }^{1}$ http://rise.com.br
} 
um grupo de 20 clientes, que validariam os requisitos da aplicação durante o uso da versão beta;

- Os clientes deveriam ter a possibilidade de contratar e usar a aplicação sem nenhuma intervenção manual da empresa provedora do serviço;

- Cada cliente deveria poder gerenciar seus próprios usuários;

- A aplicação iria executar em um ambiente virtualizado com 613MB de memória e 30GB de armazenamento;

- Os requisitos da aplicação deveriam ser levantados e implementados de forma incremental;

- A aplicação deveria aproveitar o máximo de recursos possível.

A necessidade de prover uma aplicação web, que pudesse ser contratada pelo cliente e paga por uso, sem a intervenção do provedor de serviço fortaleceram a escolha da abordagem SaaS. Adicionalmente, os recursos limitados de hardware e a necessidade de prover a mesma aplicação para 20 clientes exige o máximo de otimização no uso do hardware. Dentre os 3 tipos de SaaS apresentados na seção anterior, multitenancy apresentou-se como a melhor escolha.

\section{METODOLOGIA}

Para conduzir a etapa de desenvolvimento de um aplicativo na indústria seguiremos o processo definido na Figura 1. De acordo com a figura, a primeira etapa consiste em definir uma arquitetura do aplicativo a ser desenvolvido. A segunda etapa foi escolher as tecnologias que seriam utilizadas para a implementação dos requisitos funcionais e não funcionais da aplicação, além da migração dos dados já cadastrados que anteriormente eram manipulados por uma aplicação legada, utilizada por um dos clientes iniciais da aplicação. Durante a etapa de implementação foi adotado SCRUM[1] como metodologia de desenvolvimento, o que facilitou a realização de releases incrementais e rápidos. Por fim, na terceira etapa é realizado a coleta de feedback dos usuários, de forma que seja possível melhorar a aplicação no tocante a requisitos funcionais e não-funcionais. Uma sequência de vários ciclos compostos da etapa de "implementação" e "validação da aplicação" é realizado com o objetivo de proporcionar uma evolução incremental do produto.

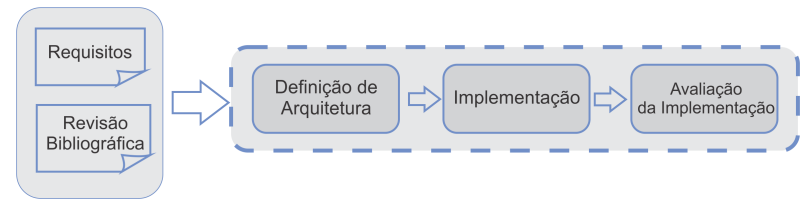

Figure 1: Metodologia Utilizada para Desenvolvimento de Aplicação na Indústria.

\section{DEFINIÇÃO DE ARQUITETURA}

A separação de interesses entre requisitos relacionados ao negócio principal da aplicação e requisitos inerentes a multitenancy também é um fator crítico que deve ser levado em consideração. A aplicação deveria ser implementada de forma que o código de regra de negócio sofresse o mínimo de interferência possível do código associado a implementações de multi-tenancy.

Estes problemas podem ser superados integrando cuidadosamente multi-tenancy na arquitetura. No restante desta seção, descrevemos os componentes da arquitetura abordada por Bezemer et al. [2] para implementação de multi-tenancy como um interesse transversal.

Os principais componentes da arquitetura definida por Bezemer são:

Autenticação Pelo motivo de uma aplicação multi-tenancy ter apenas uma instância da aplicação e do banco de dados, todos os tenants usam o mesmo ambiente físico. A fim de oferecer a customização do ambiente e ter certeza de que os tenants podem acessar somente os seus próprios dados, tenants devem ser autenticados. Como a autenticação de usuário, normalmente, já esta presente na aplicação de destino, um mecanismo específico para autenticação de tenants faz-se necessário por duas razões: (1) geralmente é mais fácil introduzir um mecanismo de autenticação adicional, do que mudar um já existente; e (2) a autenticação de tenants permite que um único usuário faça parte de uma ou mais organizações lógicas, o que estende a idéia de autenticação com grupo de usuários.

Configuração Em uma aplicação multi-tenancy a customização deve ser possível através de configuração. A fim de permitir que o usuário tenha uma experiência como se ele estivesse trabalhando em um ambiente dedicado, é necessário permitir pelo menos os seguintes tipos de configuração: estilo de Layout ,configurações gerais definidas pelo usuário, configurações de localização de arquivos e configurações de workflows.

Banco de Dados Em uma aplicação multi-tenancy há uma grande exigência para o isolamento dos dados. Como todos os tenants usam a mesma instância de um banco de dados é necessário ter certeza de que eles podem acessar somente seus próprios dados. Atualmente sistemas de gerenciamento de dados (Data Base Management Systems - DBMS) de prateleira não são capazes de lidar com multi-tenancy, isso deve ser feito em uma camada entre a camada lógica de negócios e o pool de banco de dados de aplicações. As principais tarefas dessa camada são as seguintes: criação de novos tenants no banco de dados, adaptação de consultas executadas no SGBD e balanceamento de carga.

\subsection{Tecnologias}

Após a escolha de uma arquitetura de referência para ser adotada por nossa aplicação, tem-se a necessidade de escolher as tecnologias que serão adotadas para a implementação. Durante a escolha dessas tecnologias avaliou-se a possibilidade do uso de tecnologias existentes na plataforma Java, como $\mathrm{JSF}^{2}$, Struts $2^{3}$, Spring Framework ${ }^{4}$ e Grails ${ }^{5}$. A escolha da plataforma Java deve-se ao fato da mesma ser bem consolidada tanto em ambiente acadêmico quanto no mercado. A partir de reuniões com os envolvidos observou-se que Grails apresentava um conjunto de recursos que poderiam dar produtividade para a equipe de desenvolvimento que não foram encontrados nas outras tecnologias como geração de CRUD (Create, Read, Update e Delete), arquitetura baseada em plugins que permite a criação e reuso

\footnotetext{
$\overline{{ }^{2} \text { http://javaserverfaces.java.net }}$

${ }^{3}$ http://struts.apache.org/2.3.1.2/index.html

${ }^{4}$ http://www.springsource.org/spring-framework

${ }^{5}$ http://grails.org
} 
de componentes, e total integração com frameworks e APIs Java.

O Grails é um framework web open-source que utiliza a linguagem Groovy ${ }^{6}$, e outros frameworks consagrados como Hibernate $^{7}$, Spring Framework e Sitemesh ${ }^{8}$. Grails propõese a trazer a produtividade do Ruby on Rails para a plataforma Java, porém ele possui uma grande vantagem, já que é baseado na linguagem Groovy.

A aplicação que está sendo desenvolvida, em particular se beneficiará da capacidade de definir métodos e propriedades em tempo de execução, disponibilizado pela linguagem Groovy. Essa característica da linguagem Groovy é chamada de metaprogramação. Em uma linguagem estática como java, o acesso a uma propriedade ou invocação de um método é resolvido em tempo de compilação. Em comparação, Groovy não resolve o acessos à propriedades ou invocação de métodos até que a aplicação seja executada[8]. Uma aplicação que utiliza essa linguagem pode dinamicamente definir métodos ou propriedades em tempo de execução, isso vai de encontro à necessidade de customização das aplicações, dado que com a utilização desse recurso pode-se adicionar atributos e customizar comportamentos de um tenant específico futuramente.

Outro ponto que influenciou bastante na escolha de Grails foi sua arquitetura baseada em plugins. Grails não se propões a ter todas as respostas e soluções para o desenvolvimento de aplicações web. Ao invés disso, ele provê uma arquitetura baseada em plugins e um repositório mantido pela comunidade de desenvolvedores onde é possível encontrar plugins que implementam as mais diversas funcionalidades como segurança, teste, busca, geração de relatórios, etc. Essa abordagem proporciona o reuso e permite que funcionalidades de difícil implementação possam ser adicionadas na aplicação de forma bastante simples.

Além das tecnologias relacionadas à programação, foi necessário escolher a tecnologia utilizada para armazenamento de dados. Grails Framework permite optar por qualquer SGBD relacional para armazenar dados. A opcão escolhida foi o SGBD PostgreSQL.

\subsection{Prototipagem}

A partir da escolha da arquitetura de referência, das tecnologias a serem adotadas e da divisão dos módulos da aplicação, chegou-se a proposta de arquitetura apresentada na Figura 2. A proposta da aplicação possui 5 módulos associados à lógica de negócio da aplicação, esses módulos foram descritos anteriormente na seção 2. Foi adotado o Framework Grails para auxílio ao desenvolvimento da aplicação web e juntamente com ele foram adotados alguns de seus plugins. Para validar arquitetura proposta foi desenvolvido um protótipo contendo uma parte pequena dos requisitos funcionais da aplicação. Nesse protótipo serão implementados os cadastros de Projeto, Módulo e Features. A implementação do protótipo tem o objetivo de verificar a viabilidade de implementação e o atendimento dos objetivos da arquitetura.

Com o objetivo de implementar os requisitos multi-tenancy de forma reutilizável, a solução foi implementar esses requisitos como um plugin do Grails, dessa forma aplicações futuras poderiam se beneficiar do código implementado. Uma vantagem do uso dessa abordagem é que dentro de um plugin

\footnotetext{
${ }^{6}$ http://groovy.codehaus.org

${ }^{7}$ http://www.hibernate.org

${ }^{8}$ http://www.sitemesh.org
}

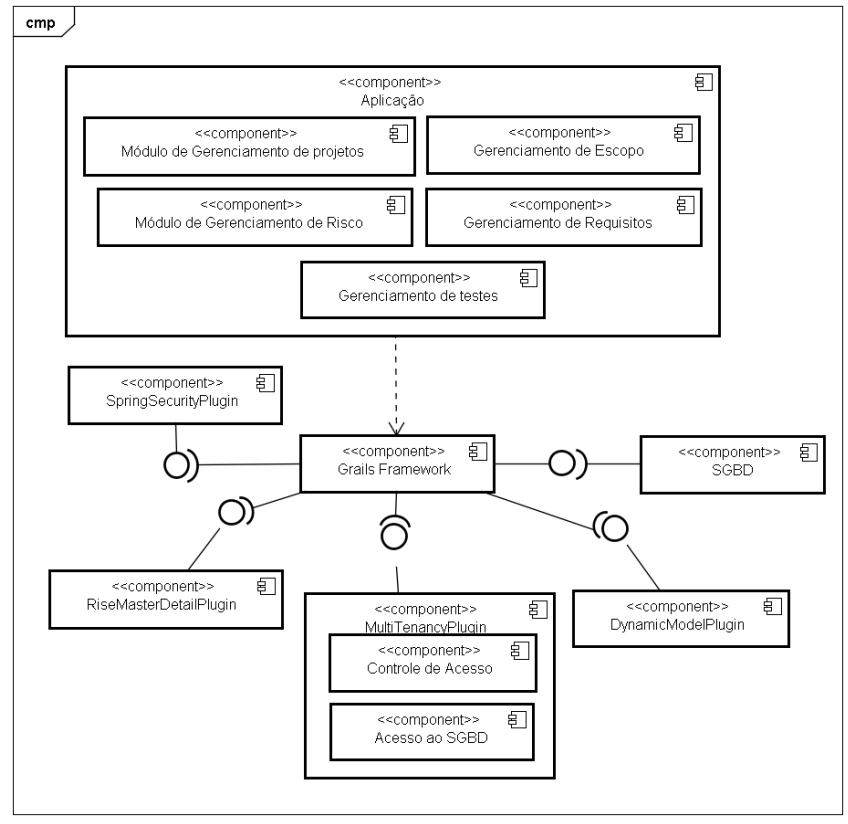

Figure 2: Arquitetura da Aplicação

grails é possível adicionar não só classes implementadas em Groovy ou Java, mas também páginas da camada de visão e outros tipos de arquivo. Antes de implementar os requisitos multi-tenancy como plugin foi realizado uma pesquisa no repositório de plugins do Grails para verificar se já existia algo semelhante ao que pretendia-se implementar.

Durante a pesquisa foi encontrado o plugin Multi-Tenant Core ${ }^{9}$ que implementa as funcionalidades de 2 dos componentes mencionados na arquitetura de referência [2], o componente de autenticação e o componentes de acesso ao banco de dados. Esse plugin implementa a funcionalidade de associar um usuário da aplicação a um tenant específico e, além disso, realiza de forma dinâmica o filtro dos dados durante uma consulta ao banco de dados, de forma que um usuário da aplicação tenha acesso apenas aos dados vinculados ao seu tenant. A autenticação e controle de acesso pode ser realizado através da integração desse plugin com o Spring Security ${ }^{10}$, um framework de controle de acesso bastante utilizado em aplicações Java.

O terceiro componente mencionado na arquitetura proposta, até então não implementado, é o componente de configuração. O objetivo desse componente é gerenciar as configurações relacionadas a cada tenant e prover funcionalidades de customização da aplicação para cada tenant. Essa customização pode ir desde alterações em interface com o usuário até alteração nas classes de negócio, tudo isso realizado de forma dinâmica. Um outro plugin encontrado Durante a pesquisa foi o plugin Dynamic Domain Class $^{11}$ que proporciona a criação de classes de domínio de forma dinâmica, esse plugin ajudou a validar a idéia de usar Groovy e Grails para customizar as classes de domínio em tempo de execução.

\footnotetext{
${ }^{9}$ http://multi-tenant.github.com/grails-multi-tenant-core

${ }^{10}$ http://static.springsource.org/springsecurity/site/index.html

${ }^{11} \mathrm{http}: / /$ code.google.com/p/grails-dynamic-domain-classplugin/
} 


\begin{tabular}{|c|c|}
\hline ATRIBUTO & AVALIAÇĀO \\
\hline$\overline{\text { Disponibilidade }}$ & $\begin{array}{l}\text { Como foi adotado um SGBD PostgreSQL, é pos- } \\
\text { sivel agendar tarefas automáticas no servidor } \\
\text { para executar backups periódicos. }\end{array}$ \\
\hline Integridade Conceitual & $\begin{array}{l}\text { Tanto as entidades de negócio quanto as classes } \\
\text { utilitárias da aplicação foram modularizadas em } \\
\text { plugins de forma que os interesses da aplicação } \\
\text { foram bem definidos e separados. }\end{array}$ \\
\hline Flexibilidade & $\begin{array}{l}\text { A flexibilidade pode ser alcançada através do } \\
\text { uso das característica de linguagem dinâmica } \\
\text { existente na linguagem Groovy. Dessa forma } \\
\text { é possivel criar Entidade de domínio e alterar } \\
\text { entidades já existentes em tempo de execução, } \\
\text { caso seja necessário. }\end{array}$ \\
\hline Interoperabilidade & $\begin{array}{l}\text { O uso de plugins Grails permite que os dados da } \\
\text { aplicação possam ser expostos através de REST } \\
\text { ou Web Services para os usuários. }\end{array}$ \\
\hline Manutenibilidade & $\begin{array}{l}\text { A arquitetura de plugins do Grails facilita a ma- } \\
\text { nutenção dos código já que cada plugin pode } \\
\text { ser mantido separadamente, reduzindo as de- } \\
\text { pendências entre os componentes da aplicação. }\end{array}$ \\
\hline Gerenciabilidade & $\begin{array}{l}\text { Não foi possivel identificar soluções para esse } \\
\text { atributo durante a implementação do protótipo. }\end{array}$ \\
\hline Performance & $\begin{array}{l}\text { Não foi possivel identificar soluções para esse } \\
\text { atributo durante a implementação do protótipo. }\end{array}$ \\
\hline Reusabilidade & $\begin{array}{l}\text { As partes da aplicação implementadas como } \\
\text { plugins podem ser reutilizadas em aplicações fu- } \\
\text { turas facilmente, sem a necessidade de qualquer } \\
\text { adaptação no plugin. }\end{array}$ \\
\hline Escalabilidade & $\begin{array}{l}\text { Não foi possivel identificar soluções para esse } \\
\text { atributo durante a implementação do protótipo. }\end{array}$ \\
\hline Segurança & $\begin{array}{l}\text { A autenticação e controle de acesso puderam ser } \\
\text { garantidos pelo uso do Framework Spring Secu- } \\
\text { rity, que pôde ser facilmente integrado com o } \\
\text { plugin de multi-tenancy no atendimento do re- } \\
\text { quisito de isolamento de acesso entre dados dos } \\
\text { tenants. }\end{array}$ \\
\hline Testabilidade & $\begin{array}{l}\text { O Grails Framework provê uma infraestrutura } \\
\text { para auxiliar na implementação de testes unitá- } \\
\text { rios que utiliza JUnit, um framework de teste } \\
\text { para aplicaçóes java. Durante a criação de uma } \\
\text { classe o Grails Framework já cria uma classe de } \\
\text { testes correspondente para otimizar o tempo de } \\
\text { desenvolvimento. }\end{array}$ \\
\hline Usabilidade & $\begin{array}{l}\text { Grails trabalha com templates para geração de } \\
\text { telas. Embora já possua um template padrão, } \\
\text { o desenvolvedor pode implementar um template } \\
\text { próprio que atenda à necessidades específicas de } \\
\text { interação com o usuário. O template padrão do } \\
\text { Grails Framework já implementa alguns padróes } \\
\text { de usabilidade bem estabelecidos no mercado. }\end{array}$ \\
\hline
\end{tabular}

Table 1: Resultado da Aplicação dos Critérios de Exclusão

Durante a implementação detectou-se que o padrão de geração de telas do Grails não satisfazia aos anseios dos usuários quanto à forma de interação com o aplicativo. A partir dessa necessidade foi desenvolvido um plugin para implementar o padrão de tela Mestre/Detalhe [7], não implementado nativamente pelo gerador de telas padrão do Grails. Durante a implementação desse plugin identificou-se que poderia ser viável implementar módulos completos da aplicação como plugins Grails, desde classes de negócio à camada de visão.

Após a implementação do protótipo foi realizada a avaliação do protótipo para verificar quais atributos de qualidade desejáveis foram atendidos. A Tabela 1 apresenta cada atributo de qualidade e uma breve descrição de como ele foi atendido.

Após a avaliação do protótipo foi possível observar que a solução proposta atendia a 8 dos 12 atributos de qualidade desejados. Quatro atributos de qualidade (disponibilidade, gerenciabilidade, performance, escalabilidade) não puderam ser avaliados durante a implementação do protótipo inicial. A avaliação foi considerada satisfatória, considerando que os quatro atributos de qualidade da arquitetura que não foram atendidos inicialmente não geraram nenhum impedimento para a geração da primeira versão do aplicativo. Ao fim da implementação do protótipo deu-se início ao desenvolvimento da aplicação real. Ao final da implementação da primeira versão da aplicação, foi necessário criar algum mecanismo para migrar os dados já cadastrados pela aplicação legada.

\section{CONCLUSÕES}

Esse trabalho apresentou uma abordadgem para implementação de aplicações multi-tenancy que utiliza uma arquitetura de plugins e o recurso de metaprogramação, existente em várias linguagens dinâmicas. A arquitetura de plugins permitiu a modularização dos requisitos de forma que os artefatos (plugins) possam ser reutilizados em aplicações futuras. Já o uso de metaprogramação, permitiu a separação de interesses durante a implementação. Dessa forma os requisitos multi-tenancy puderam ser inseridos na aplicação de forma pouco invasiva. A separação de interesses provida pela abordagem proposta permitiu um alto grau de reuso durante o desenvolvimento da aplicação. Como trabalhos futuros serão realizados experimentos que validem essa abordagem em um ambiente com grande número de requisições e manipulação de grande massa de dados.

\section{REFERENCES}

[1] P. Agarwal. Continuous scrum: agile management of saas products. In Proceedings of the 4th India Software Engineering Conference, ISEC '11, pages 51-60, New York, NY, USA, 2011. ACM.

[2] C.-P. Bezemer, A. Zaidman, B. Platzbeecker, T. Hurkmans, and A. 't Hart. Enabling multi-tenancy: An industrial experience report. In Software Maintenance (ICSM), 2010 IEEE International Conference on, pages $1-8$, sept. 2010.

[3] Y. C. Cavalcanti, I. Do Carmo Machado, P. A. Da Mota, S. Neto, L. L. Lobato, E. S. De Almeida, and S. R. De Lemos Meira. Towards metamodel support for variability and traceability in software product lines. Proceedings of the 5th Workshop on Variability Modeling of SoftwareIntensive Systems VaMoS 11, (840):49-57, 2011.

[4] F. Chong and G. Carraro. Architecture strategies for catching the long tail. 2006.

[5] Gartner. Gartner says worldwide software-as-a-service revenue to reach 14.5 billion in 2012, 2012.

[6] I. S. Harris and Z. Ahmed. An open multi-tenant architecture to leverage smes. European Journal of Scientific Research, 65(4):601-610, 2011.

[7] T. Neil. 12 standard screen patterns, 2010.

[8] C. Richardson. Orm in dynamic languages. Commun. ACM, 52(4):48-55, Apr. 2009.

[9] Z. H. Wang, C. J. Guo, B. Gao, W. Sun, Z. Zhang, and W. H. An. A study and performance evaluation of the multi-tenant data tier design patterns for service oriented computing. In Proceedings of the 2008 IEEE International Conference on e-Business Engineering, pages 94-101, Washington, DC, USA, 2008. IEEE Computer Society. 